This is a self-archived - parallel published version of this article in the publication archive of the University of Vaasa. It might differ from the original.

\title{
Co-optimization of transmission switching and variable reactance devices control under worst- case wind uncertainty
}

Author(s): Nikoobakht, Ahmad; Aghaei, Jamshid; Lotfi, Mohamed; Osório, Gerardo J.; Shafie-khah, Miadreza; Catalão, João P. S.

Title: Co-optimization of transmission switching and variable reactance devices control under worst-case wind uncertainty

Year: $\quad 2019$

Version: Accepted manuscript

Copyright C2019 IEEE. Personal use of this material is permitted. Permission from IEEE must be obtained for all other uses, in any current or future media, including reprinting/republishing this material for advertising or promotional purposes, creating new collective works, for resale or redistribution to servers or lists, or reuse of any copyrighted component of this work in other works.

\section{Please cite the original version:}

Nikoobakht, A., Aghaei, J., Lotfi, M., Osório, G.J., Shafie-khah, M., \& Catalão, J.P.S., (2019). Co-optimization of transmission switching and variable reactance devices control under worstcase wind uncertainty. In: IEEE Conference on Control Technology and Applications (CCTA), Hong Kong, China (pp. 86-91). https://doi.org/10.1109/CCTA.2019.8920607 


\title{
Co-optimization of Transmission Switching and Variable Reactance Devices Control under Worst-Case Wind Uncertainty
}

\author{
Ahmad Nikoobakht, Jamshid Aghaei, Senior Member, IEEE, Mohamed Lotfi, Member, IEEE, \\ Gerardo J. Osório, Miadreza Shafie-khah, Senior Member, IEEE, and João P. S. Catalão, Senior Member, IEEE
}

\begin{abstract}
The work reported in this paper jointly addresses two major challenges in modern power systems: 1) systematically maximizing wind power generation (WPG) utilization under worst-case uncertainty and 2) employing mixed integer-nonlinear programming (MINLP) in the co-optimization of variable reactance devices (VRD) and transmission switching (TS) in an AC optimal power flow problem (ACOPF). The first challenge is solved by proposing an interval based robust approach to identify the worst-case WPG uncertainty. Similarly, to overcome the second challenge, a tri-level decomposition algorithm is used to decompose the MINLP representation into one consisting of one mixed-integer linear programming (MILP) and two nonlinear programming (NLPs) problems. Finally, the effectiveness and efficiency of the proposed model is shown by analysing results from testing on the modified RTS-96 system.
\end{abstract}

\section{NOMENCLATURE}

\section{A. Indices}

$w, g \quad$ Wind farm and Generation units.

$n / m \quad$ System buses.

$k: \quad$ Transmission line.

$(\cdot)^{ \pm}$: Wind uncertainty (“+”upper and “-” lower boundaries of possible wind uncertainty).

Note that $(\cdot)^{0}$ relate to the first stage and $(\cdot)^{ \pm}$relate to the second stage variable (uncertainty condition).

\section{B. Sets}

$\Omega_{k}^{V R D} \quad$ Set of transmission lines with VRD.

$\chi_{n} \quad$ Set of generation units connected to bus $n$.

$\Omega_{n} \quad$ Set of transmission lines connected to bus $n$.

\section{Continuous Variables}

$\delta_{n m}^{(\cdot)} \quad$ Difference of voltage angles across line $(n, m)$.

$P_{g}^{(\cdot)} / Q_{g}^{(\cdot)} \quad$ Real/ reactive generation of unit $g$.

$W_{w}^{(\cdot)} \quad$ Forecasted generation of wind farm $w$.

$V_{n}^{(\cdot)} \quad$ Voltage magnitude at bus $\mathrm{n}$.

$\underline{\aleph}_{n}^{n} / \overline{\boldsymbol{\aleph}}_{n} \quad$ Slack variables.

The work of J.P.S. Catalão was supported by FEDER funds through COMPETE 2020 and by Portuguese funds through FCT, under POCI-01-0145-FEDER-029803 (02/SAICT/2017).

Ahmad Nikoobakht is with the Higher Education Center of Eghlid, Eghlid, Iran (a.nikoobakht@eghlid.ac.ir).

Jamshid Aghaei is with the Shiraz University of Technology, Shiraz, Iran (aghaei@sutech.ac.ir).

Mohamed Lotfi and João P. S. Catalão are with INESC TEC and FEUP, Porto, Portugal (mohd.f.lotfi@gmail.com; catalao@fe.up.pt).

Gerardo J. Osório is with C-MAST, UBI, Covilha, Portugal (gjosilva@gmail.com).

Miadreza Shafie-khah is with the University of Vaasa, Vaasa, Finland (miadreza@gmail.com).
$P_{n m}^{(\cdot)} / Q_{n m}^{(\cdot)} \quad$ Active/reactive power flow from bus $n$ to bus $m$.

$U s_{g}^{ \pm} / D s_{g}^{ \pm} \quad$ Up and down spinning reserve for unit $g$.

$\tilde{x}_{k}^{(\cdot)} \quad$ Reactance of the VRD installed on line $k$.

$G_{k} / B_{k} \quad$ Conductance/ series admittance of line $k$.

$\Theta / \bar{\Theta} \quad$ Total cost for base case/uncertainty case.

$\alpha \quad$ Variation of wind uncertainty.

$\ell_{(\bullet)}^{(\bullet)} \quad$ Dual variable.

$\mathfrak{R} \quad$ Active power mismatch.

\section{Binary Variables}

$z_{k} \quad$ Switching state of line $k$.

$u_{g} \quad$ Status of generating unit $\mathrm{g}$.

\section{E. Constants}

$\delta_{k}^{\min } / \delta_{k}^{\max } \quad \operatorname{Min} / \max$ difference of voltage angle across line $k$. $V_{n}^{\min } / V_{n}^{\max } \quad \operatorname{Min} / \max$ voltage magnitude at bus $n$.

$\tilde{x}_{k}^{\min } / \tilde{x}_{k}^{\max } \quad \mathrm{Min} / \max$ reactance change of the TCSC for line $k$.

$\kappa_{g} / \kappa_{g}^{s r} \quad$ Cost of wind uncertainty / normal conditions of generation unit $g$.

$P_{g}^{\min } / P_{g}^{\max } \quad \mathrm{Min} / \mathrm{max}$ active power generation of unit $g$.

$Q_{g}^{\min } / Q_{g}^{\max } \quad \mathrm{Min} / \max$ active power generation of unit $g$.

$U s_{g}^{\max } / D s_{g}^{\max }$ Max up/down spinning reserve for unit $g$.

$S_{k}^{(-)} \quad$ Maximum amount of MVA flow through line $k$

$\bar{W}_{w} \quad$ Forecasted power generation of wind farm $w$.

$L_{n}^{P} / L_{n}^{Q} \quad$ Active and reactive power consumption via demand

$n$.

$K \quad$ A large number (positive).

$B_{k 0} \quad$ Shunt admittance of transmission line $k$.

$\vartheta_{k} \quad$ Binary variable implying whether VRD is placed on line $k$ or not.

$\lambda \quad$ Compensation level of VRD susceptance.

$\eta \quad$ Number of transmission lines that are allowed to be opened in a system.

\section{INTRODUCTION}

Over the last decade, the share of wind power generation (WPG) in power systems has been growing significantly. However, the inherent uncertainty of WPG and in addition to technical constraints due to congestion of transmission lines pose limitations on their large-scale integration into power systems. Due to the low cost of WPG, it is essential to seek approaches that help overcome these limitations and improve transfer capability. For this, researchers have utilized numerous different approaches to boost the transfer capability over existing transmission networks. Demand-side management strategies, including the use of energy storage systems, can effectively enhance transfer capability and improve integration of the WPGs. 
However, the potential of these non-conventional assets is limited by the lack of flexible load and the high cost of energy storage systems. Alternatively, the other way to alleviate the transmission limitation is to develop the transmission grid via constructing new transmission lines. Nevertheless, constructing a new transmission line necessitates a long permitting lead time and is capital intensive. Therefore, it is necessary to use the current transmission grid effectively in order to increase the power transfer capability (of or through) the existing transmission grid [1]. The two modern technologies that would improve transfer capability are based on variable reactance devices (VRDs) and transmission switching (TS) actions. Accordingly, this work focuses on the VRDs, which can comprise Thyristor-Controlled Series Compensator (TCSCs) or the more recent technology Smart Wire device (SWD) [1], [2]. The VRDs or TCSC devices and SWD, are power electronic devices that can be used to modify the reactance of a specific part of transmission lines. As a result, the power transfer capability of the transmission grid can be increased. The VRD is an efficacious tool to enhance transfer capability of the transmission grid. In [3], an AC Optimal Power Flow (ACOPF) model was used to increase the available transfer capacity of the transmission grid using TCSC devices. Here the TS action means the change of the transmission grid topology by closing or opening transmission lines. Improving the power flow capacity of a transmission grid through TS action has received significant attention in the literature (refer to [4] and [5]). Hence, the TS operation is a low-cost power flow control approach that would enhance integration of intermittent WPGs and reduce the system costs. Previous studies [3], [6], [7] and [8] have investigated the separate effects of TS action and VRDs in the OPF problem with WPG uncertainty.

Many researchers have utilized the simpler direct current OPF (DCOPF) model (which does not account for variations in voltage magnitude, reactive power and network losses) to model VRDs and TS action in power systems. The DCOPF was used only due to its speed and simplicity. It is often overlooked that modelling TS action and VRDs with DCOPF equations is inacceptable for the following reasons:

1) DCOPF neglects reactive power and transmission losses and may thereby lead to an infeasible solution. In this case the security of the power system may be endangered, especially when implementing the resulting TS decisions. Similarly, the system stability is directly related to the reactive power adequacy in the power system. [7]

2) Other potential benefits of TS actions and VRDs cannot be utilized in the DCOPF model. Namely, reduction of the power grid losses or modifying voltage violations cannot be utilized [7], [9]

Accordingly, it is thus essential to address this question: "How much of the renewable uncertainty can be mitigated by integration of both TS action and VRD in an ACOPF problem?" It is of special interest to find out the worst-case renewable uncertainty with these power flow control technologies (PFCTs). To properly answer this question, this paper incorporates both TS actions and VRD into an ACOPF problem with worst-case renewable uncertainty.
It must be remembered that incorporating TS and VRD into a full AC-OPF involves the modeling of binary variables, which results in a mixed integer non-linear programming (MINLP) problem: a very complex one to solve in reasonable time. Accordingly, to solve this challenge a tri-level benders decomposition (BD) approach is implemented to decompose the problem into a master one with the network-constrained DC-OPF, which is a mixedinteger linear programming (MILP) problem, and two network-constrained AC-OPF sub-problems, which are NLP problems. The global optimal solution for the proposed model can be guaranteed by using commercially available software and solvers.

Another question posed by a major challenge faced by a system operator is: "how to model the worst-case WPG uncertainty?" Several models currently exist for the modeling of WPG uncertainty. Stochastic approach (SA) and standard robust optimization (SRO) approach are currently the most popular approaches for the modeling of WPG uncertainty. Every method has its own pros and cons.

For example, recently a considerable volume of literature has emerged addressing the utilization of SA which describes the uncertain parameters by means of scenarios [4]. Therefore, the analysis of SA in particular was problematic because this approach requires a large number of scenarios to model the uncertain parameter which results in computational intractability if large systems are considered. In this way, in recent years, researchers have shown an increased interest in the SRO approach such as [10], [11] and [12].

An often overlooked point is that for the SRO approach the variant range of WPG uncertainty needs to be known $a$ priori, and the lower and upper horizons of uncertainty should be fixed before solving the problem [13]. Accordingly, this necessity is a main disadvantage of the SRO approach, due to worst-case WPG uncertainty which cannot be identified.

Thus, to address the above concern, this paper proposes an interval-based robust (IBR) approach to identify worse-case WPG uncertainty. This method does not require the large number of scenarios and is not computationally expensive compared to SA. It also does not require to predefine variant range of the WPG uncertainty. The objective of the IBR approach is maximizing the acceptable horizon of uncertainty to identify worse-case WPG uncertainty while satisfying a predetermined cost threshold.

Another advantage of using IBR approach is that it can be solved directly for a MILP problem, while the SRO approach cannot be solved directly. [10], [11] To clarify, in order to solve the SRO approach the column-and-constraint generation algorithm [14] are used. The main disadvantage of the column-and-constraint generation algorithm is that the upper and lower bounds of worst case uncertainty cannot be obtained simultaneously through this approach. Nevertheless, the proposed IBR approach could be solved directly for the MILP problems or could be solved by a general benders decomposition that does not need to optimality cuts. In fact, the general benders decomposition can be converged just by feasibility cuts. 
Accordingly, the main novelty of the approach and study presented are as follows:

- A new interval-based robust method is proposed to identify worse-case uncertainty. Under the proposed robust framework, the largest variant range of uncertainty that the system can accommodate is evaluated.

- The interval-based robust method is introduced for the ACOPF model. The proposed model co-operation the TS action and VRDs, simultaneously, to enhance utilization of WPG.

\section{MATHEMATICAL ACOPF FORMULATION WITH PFCTS}

In this section, all constraints implemented in the original ACOPF problem with PFCTs model are described in detail. Accordingly, the equation of the ACOPF problem with PFCT models is as follows:

$\min \Theta=\sum_{g}\left(\kappa_{g} P_{g}+\kappa_{g}^{s r}\left(U s_{g}^{ \pm}+D s_{g}^{ \pm}\right)\right)$

Equation (1) is objective function or total cost (TC) of the proposed problem, which included two key parts: first and second stage parts. The first stage part is related to cost of generation units at the normal condition (or base case condition), and the second stage part is related to the cost of up and down-spinning reserves to cover to cover the upper and lower boundaries of worst-case wind power uncertainty.

$$
\begin{aligned}
& \sum_{g \in \chi_{n}} P_{g}+W_{w}-L_{n}^{P}=\sum_{m \in \Omega_{n}} P_{n m}, \quad \forall W_{w}=\bar{W}_{w} \\
& \sum_{g \in \chi_{n}} Q_{g}-L_{n}^{Q}=\sum_{m \in \Omega_{n}} Q_{n m}
\end{aligned}
$$

The active and reactive power balance prior to worstcase wind uncertainty realization has been denoted by Constraints (2) and (3).

$$
\begin{aligned}
& P_{n m}=\left(G_{k} V_{n}^{2}-V_{n} V_{m}\left(G_{k} \cos \delta_{n m}+B_{k} \sin \delta_{n m}\right)\right) \cdot z_{k} \\
& Q_{n m}=-\left(\left(B_{k}+B_{k 0}\right) V_{n}^{2}+V_{n} V_{m}\left(B_{k} \cos \delta_{n m}-G_{k} \sin \delta_{n m}\right)\right) \cdot z_{k} \\
& \left\{\begin{array}{l}
G_{k}=\left(\frac{r_{k}}{r_{k}^{2}+\left(x_{k}-\vartheta_{k} \cdot \tilde{x}_{k}\right)^{2}}\right) \\
B_{k}=\left(\frac{\left(x_{k}-\vartheta_{k} \cdot \tilde{x}_{k}\right)}{r_{k}^{2}+\left(x_{k}-\vartheta_{k} \cdot \tilde{x}_{k}\right)^{2}}\right)
\end{array}\right.
\end{aligned}
$$

The active and reactive power flow through a transmission line with (without) VRD and switched capability. Binary variable $z_{k}$ is the indicator of switched lines, with 0 indicating line $k$ is close and $O$ is opened. Conductance and series admittance of line $k$ is shown by (4). Binary variable $\vartheta_{k}$ is used to indicate whether VRD is installed on transmission line $k$, with 0 indicating not installed and 1 indicating installed. Noted that, $z_{k}$ for the transmission lines equipped with VRD is equal to 1 , these transmission lines cannot be switched. Similarly, $\vartheta_{k}$ for the switchable transmission lines is equal to 0 . The upper and lower limits of active and reactive power generation of generator units (GUs), angles of buses and voltage magnitudes are enforced by constraints (7)-(12).

$$
\begin{aligned}
& P_{g}^{\min } u_{g} \leq P_{g} \leq P_{g}^{\max } u_{g} \\
& Q_{g}^{\min } u_{g} \leq Q_{g} \leq Q_{g}^{\max } u_{g} \\
& P_{n m}^{2}+Q_{n m}^{2} \leq z_{k} \cdot S_{k}^{2} \\
& \delta_{k}^{\min } \leq \delta_{n m} \leq \delta_{k}^{\max } \\
& V_{n}^{\min } \leq V_{n} \leq V_{n}^{\max } \\
& \tilde{x}_{k}^{\min } \lambda \leq \tilde{x}_{k} \leq \tilde{x}_{k}^{\max } \lambda \\
& P_{g}^{ \pm}=\left(P_{g}^{0}+U s_{g}^{ \pm}-D s_{g}^{ \pm}\right) \\
& \left\{\begin{array}{l}
0 \leq U s_{g}^{ \pm} \leq U s_{g}^{\max } u_{g} \\
0 \leq D s_{g}^{ \pm} \leq D s_{g}^{\max } u_{g}
\end{array}\right.
\end{aligned}
$$

(2)-(12)

Constraints (13)-(15) denote the second-stage. Where, in (13) - (15), "+"/ "-" relate to the upper/lower limits of possible wind uncertainty, respectively.

Constraint (20) links between the first-stage and secondstage conditions. In constraint (20), the output of a GU can be changed by up/down spinning reserve, i.e., $U s_{q}^{ \pm} / D s_{q}^{ \pm}$. Constraint (14) enforce the lower and upper bounds of up/down spinning reserves for a GU.

Lastly, constraints (2)-(12) in constraint (15) are similar to (5)-(8), but for the second-stage, where the variables $P_{g}, Q_{g}, W_{w}, P_{n m}, Q_{n m}$ and $\delta_{n m}$ are replaced by $P_{g}^{ \pm}, Q_{g}^{ \pm}, W_{w}^{ \pm}$, $P_{n m}^{ \pm}, Q_{n m}^{ \pm}$and $\delta_{n m}^{ \pm}$, respectively. It should be noted that, the variables $z_{k}$ and $\tilde{x}_{k}$ in constraints (2)-(15) are determine just in first-stage.

\section{WORST-CASE WPG UNCERTAINTY MODEL}

The worst-case WPG uncertainty can be is modeled as follows:

$\max \alpha$

$W_{w}^{ \pm}=(1 \pm \alpha) \cdot \bar{W}_{w}$

$\Theta \leq \bar{\Theta}$

(2) $-(15)$

The objective function (16) maximizes the uncertainty level of the WPG to find the worst-case uncertainty. The $\alpha$ value in (16), can be changed from 0 to 1 . The upper and lower boundaries of possible WPG uncertainty are equal to the WPG output forecast (i.e., $\left.\bar{W}_{w}\right)$ multiplied by $(1+\alpha)$ and $(1-\alpha)$, respectively. The total cost in (16)-(19) must be not surpassed form pre-specified cost threshold, i.e., $\bar{\Theta}$, for any realization of WPG uncertainty which is enforced by constraints (18). Noted that, the cost threshold depends on the decision makers' conservatism level.

\section{ThreE-Stage DeComposition SOLUTION APPROACH}

Point often overlooked, the original AC-OPF problem with the nonlinear VRD and TS action becomes a mixedinteger nonlinear program (MINLP). The main disadvantage of MINLP problem is that available solvers, e.g., DICOPT and BARON, for these problems cannot obtain global optimal solution with reasonable computational burden. 
To deal with this challenge, a Benders decomposition BD approach [8] is implemented here to solve the proposed MINLP problem.

As can be seen in Fig.1, the BD approach divides the problem into the master problem, sub-problem I and subproblem II. In the master problem, the economic dispatch of GUs, status (on/off) of GUs, status (open/close) of switchable lines and reactance value of the VRD with a DC security-constrained optimal power flows (DCOPF) have been specified. Noted that, this part is a MILP problem. Subproblems I and II checks possible AC security-constrained optimal power flows (ACOPF) violations of the master solution for the firs-stage and second stage constraints, respectively.

It is important to remember that sub-problems I and II are NLP problem. If the objective values of Sub-problems I and II is larger than the predefined threshold, feasibility cuts will be generated and fed backed to master problem for next iteration. The iterative process will stop when the master solution satisfies AC network security constrains of subproblems I and II. The detail formulations of the master problem and sub-problem I and II are described as follows:

\section{A. Master problem}

The master problem with the TS action and VRD in DC network security constrains are modeled as follows: $\max \alpha$

$(17)-(18)$

The first stage constraints are:

$$
\begin{aligned}
& \left\{B_{k} \delta_{n m}\left(1-\vartheta_{k}\right)+\underline{B}_{k} \delta_{n m} \vartheta_{k}-K\left(2-z_{k}-\vartheta_{k}\right) \leq P_{n m}\right. \\
& B_{k} \delta_{n m}\left(1-\vartheta_{k}\right)+\bar{B}_{k} \delta_{n m} \vartheta_{k}+K\left(2-z_{k}-\vartheta_{k}\right) \geq P_{n m} \\
& -\bar{P}_{n m} z_{k} \leq P_{n m} \leq \bar{P}_{n m} z_{k} \\
& \text { (2), (7) and (11), } \forall\left\{P_{g}, W_{w}, P_{n m}, \delta_{n m}, z_{k}\right\}
\end{aligned}
$$

The second stage constraints are:

$$
\text { (2), (7), (11), (22) and (23), } \forall\left\{P_{g}^{ \pm}, W_{w}^{ \pm}, P_{n m}^{ \pm}, \delta_{n m}^{ \pm}, z_{k}\right\}
$$

In master problem, the objective function (20) is maximized with respect to constraints (21)-(25). Constraints (21), (24) and (25) have been mentioned in sections III and IV. Constraint (22) denotes a linear DC equation for active power flow through a transmission line that it is a switchable line or equipped with the VRD. Noted that, in constraint (22) the parameter $\vartheta_{k}$ for switchable lines is zero and for a line with the VRD is one.

Likewise, variable $z_{k}$ for transmission line that equipped with the VRD is one. Constraint (22) shows active power flow in a transmission line which may be switchable. More detail explanation about constraint constraints (22) and (23) could be found in [8].

\section{B. Sub-problem I}

This sub-problem checks possible AC network security constraint violations of the master solution from the first stage. The objective function in Eq. (26) minimizes the slack variables $\underline{\aleph}_{n}$ and $\overline{\boldsymbol{\aleph}}_{n}$.

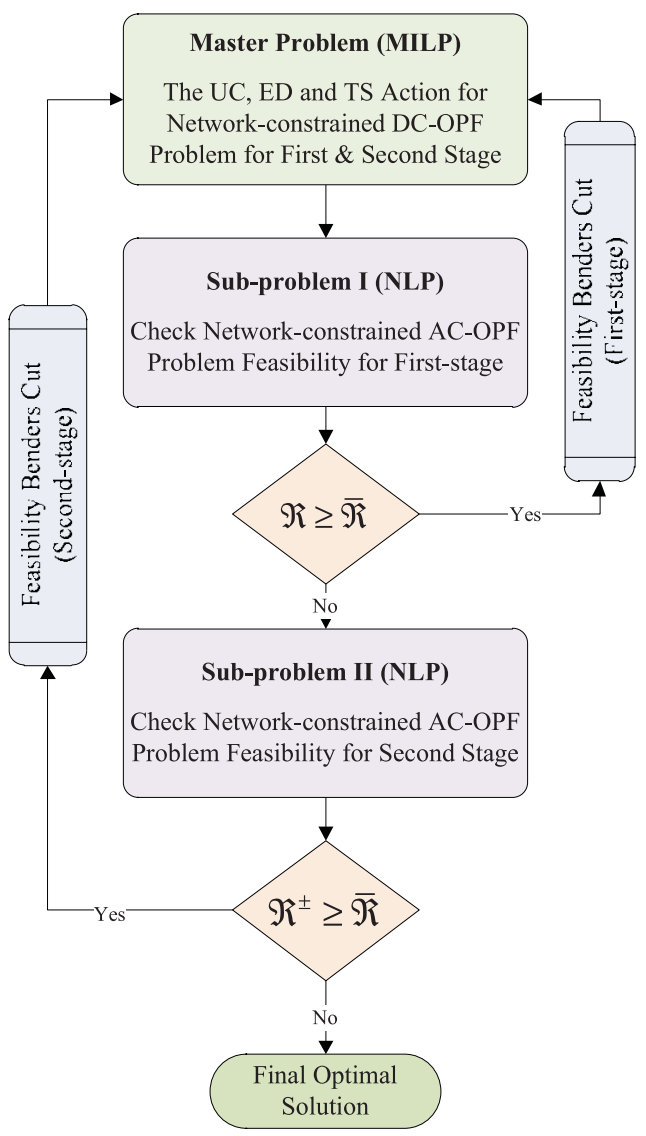

Fig.1. The solution strategy based on BD approach.

In fact, the slack variables could be considered as virtual loads/generators which are added to nodal balance constraint to mitigate mismatches, also these variables would not be zero in case that proposed master generating schedule and the DCOPF results are not able to satisfy the nodal balance.

$$
\begin{aligned}
& \min \Re=\sum_{n}\left(\underline{\aleph}_{n}+\overline{\boldsymbol{\aleph}}_{n}\right) \\
& \sum_{g \in \chi_{n}} P_{g}+W_{w}-L_{n}^{P}+\left(\overline{\boldsymbol{\aleph}}_{n}-\underline{\aleph}_{n}\right)=\sum_{m \in \Omega_{n}} P_{n m}, \quad \forall W_{w}=\bar{W}_{w} \\
& ((3),(4)-(6),(8) \text { and (19) - (12) } \\
& \left\{\forall\left\{P_{g}, W_{w}, P_{n m}, \delta_{n m}, V_{n}, z_{k}, \tilde{x}_{k}, u_{g}\right\}\right. \\
& P_{g}=\hat{P}_{g} \rightarrow \ell_{1, g} \\
& P_{n m}=\hat{P}_{n m} \rightarrow \ell_{2, k}, \forall k \in \Omega_{k}^{V R D} \\
& z_{k}=\hat{z}_{k} \rightarrow \ell_{3, k}, \forall k \notin \Omega_{k}^{V R D} \\
& u_{g}=\hat{u}_{g} \rightarrow \ell_{4, g}
\end{aligned}
$$

Constraint (28) includes the first-stage constraints. Constraint (9) fixes the values of the complicating variables to given values achieved from the master problem solution. The variable $\ell_{(\bullet)}$ in constraint (9) is dual variable that it provides sensitivity to be used in building feasibility benders cut for the master problem.

$$
\begin{aligned}
& \hat{\mathfrak{R}}+\sum_{g} \ell_{1, g}\left(P_{g}-\hat{P}_{g}\right)+\sum_{g} \ell_{4, g}\left(u_{g}-\hat{u}_{g}\right) \\
& +\sum_{k} \ell_{2, k}\left(P_{n m}-\hat{P}_{n m}\right)+\sum_{k} \ell_{3, k}\left(z_{k}-\hat{z}_{k}\right) \leq 0
\end{aligned}
$$


In this section, if $\hat{\mathfrak{R}}>\overline{\mathfrak{R}}$ the feasibility benders' cut (30) will be generated and added to the master problem to mitigate the violations (i.e., (26)) in the next iteration. $\overline{\mathfrak{R}}$ is a small positive number specified by the decision maker.

\section{Sub-problem II}

The sub-problem for second-stage is formulated as follows.

$$
\begin{aligned}
& \min \Re^{ \pm}=\sum_{n}\left(\underline{\boldsymbol{\aleph}}_{n}^{ \pm}+\overline{\boldsymbol{\aleph}}_{n}^{ \pm}\right) \\
& \left\{\begin{array}{l}
(27)-(30) \\
\forall\left\{P_{g}^{ \pm}, W_{w}^{ \pm}, P_{n m}^{ \pm}, \delta_{n m}^{ \pm}, V_{n}^{ \pm}, z_{k}, \tilde{x}_{k}, u_{g}\right\} \text { and } \\
\left\{\ell_{1, g}^{ \pm}, \ell_{2, k}^{ \pm}, \ell_{3, k}^{ \pm}, \ell_{4, g}^{ \pm}\right\}
\end{array}\right.
\end{aligned}
$$

It must be remembered that the sub-problem II, i.e., (31)(32), is similar to sub-problem I, i.e., (26)-(29) only variables $\left\{\underline{\boldsymbol{\aleph}}_{n}, \overline{\boldsymbol{\aleph}}_{n}, P_{g}, W_{w}, P_{n m}, \delta_{n m}, V_{n}, z_{k}, \tilde{x}_{k}, u_{g}, \ell_{1, g}, \ell_{2, k}, \ell_{3, k}, \ell_{4, g}\right\}$ in the Equations (26) to (29) are replaced by $\left\{\underline{\boldsymbol{\aleph}}_{n}^{ \pm}, \overline{\boldsymbol{\aleph}}_{n}^{ \pm}, P_{g}^{ \pm}, W_{w}^{ \pm}, P_{n m}^{ \pm}, \delta_{n m}^{ \pm}, V_{n}^{ \pm}, z_{k}, \tilde{x}_{k}, u_{g}, \ell_{1, g}^{ \pm}, \ell_{2, k}^{ \pm}, \ell_{3, k}^{ \pm}, \ell_{4, g}^{ \pm}\right\}$in (31)-(32), respectively. In other words, the sub-problem II checks AC network security constraint for second-stage solution of master problem. Similar to sub-problem I, if $\hat{\mathfrak{R}}^{ \pm}>\bar{\Re}$, a feasibility benders' cut similar to (30) will be generated and added to the master problem to alleviate the violations (i.e., (31)) in the next iteration. Another key point is that the variables $z_{k}$ and $u_{g}$ in sub-problems I and II are continuous variables, while they are binary variables in the original problem (16)-(19) and the master problem (20)(25). Accordingly, the sub-problems I and II convert from MINLP to NLP framework. A major advantage of NLP is that available solvers, e.g., CONOPT, for these problems can obtain global optimal solution with reasonable solution time.

\section{CASE STUdies}

To investigate the performance of the proposed intervalbased robust AC-OPF problem with and without VRD and TS action, the slightly modified RTS-96 [15] has been implemented and discussed in the following subsections. The modified RTS-96 test system has been shown in Fig. 2, which has 24 buses, 32 generators, 38 transmission lines and 1 wind farm. The peak load is $2850 \mathrm{MW}$, the share of peak load at each bus is given in [15]. The VRD have been located on Line 15-24. The minimum and maximum limits of the VRD's reactance, i.e., $\tilde{x}_{k}$, are $\tilde{x}_{k}^{\min }=-0.07$ p.u. and $\tilde{x}_{k}^{\max }=0.07$ p.u., respectively. At first, it is assumed that the wind power output at Bus 24 is $500 \mathrm{MW}$. The cost threshold is equal to $\bar{\Theta}=\$ 10850.432$. As previously mentioned, the cost threshold can be specified based on conservatism levels of decision makers. Indeed, accommodation of higher uncertain WPGs will result in higher cost thresholds. For transmission line congestion simulation, the capacity limit of some lines is reduced by $100 \mathrm{MW}$ and for other lines unchanged. The proposed problem has been performed using GAMS in a workstation PC with an Intel Core i7 processor $(4.5 \mathrm{GHz})$ with $16 \mathrm{~GB}$ RAM.

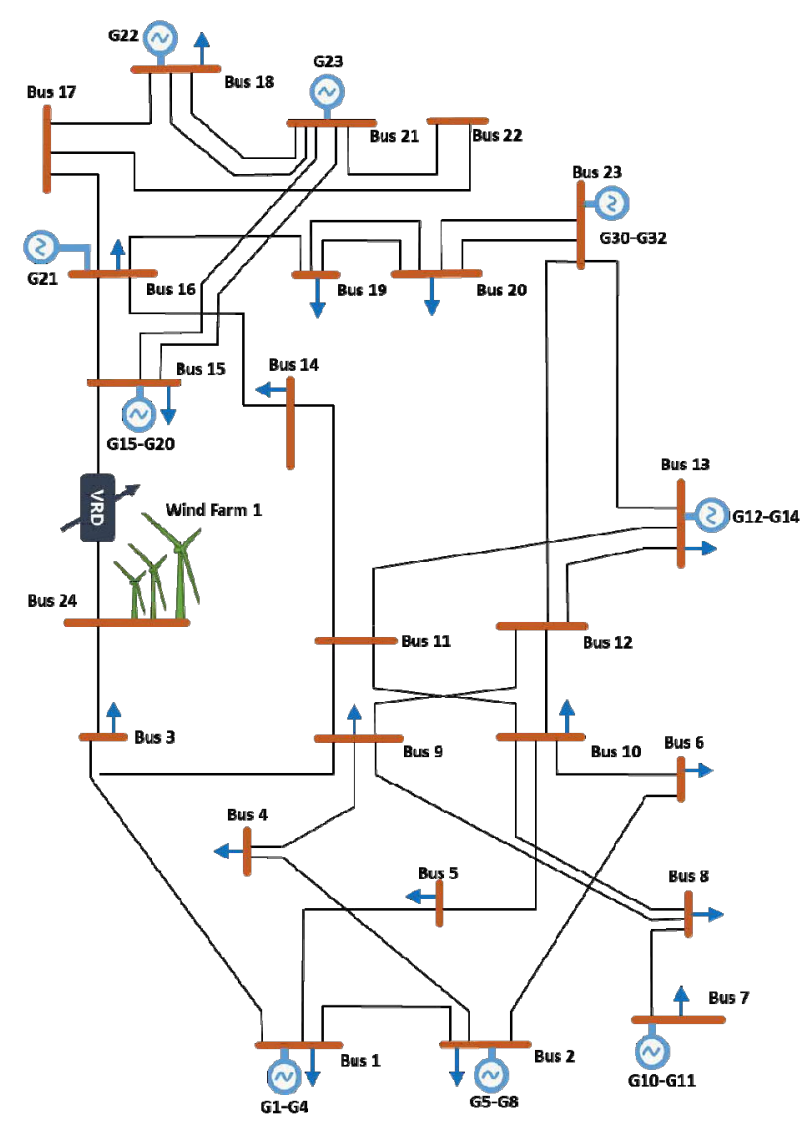

Fig. 2. The modified RTS-96 with one wind farm and VRD.

\section{A. Worst-case wind uncertainty}

Table I summarizes the solution for worst-case wind uncertainty, i.e., the $\alpha$ value without (and with) the VRD and TS action. What stands out in the table is that the $\alpha$ value for VRD is comparatively larger than that of TS action, and the value of these two cases are lower than the value for TS\&VRD. This implies that VRD and TS action are effective with respect to a case without VRD and TS action. Additionally, it is observed that a VRD leads to a better result than a TS action due to its higher capability in controlling power flows.

\section{B. Impact of Compensation Level of VRD}

Fig. 3 shows the $\alpha$ value as a function of a line's compensation level $\lambda$ and for $\lambda=7$. Interesting insights can be gleamed from the figure. (i) the $\alpha$ value is increased when $\lambda$ value for VRD increases. (ii) the $\alpha$ value for TS action is more increased when $\lambda$ value increases. This result was expected. However, it is probable that the reason for this is that the VRD at line 15-24 substantially increases the power transfer in that line.

TABLE I. OPTIMAL SOLUTION OF THE $\alpha$ VALUE UNDER LOCATIONALLOCATION MODEL FOR $\lambda=0.25$ AND $\eta=7$.

\begin{tabular}{|c|c|c|c|c|}
\hline Model & NO VRD\&TS & VRD & TS & TS\&VRD \\
\hline DCOPF & 0.022 & 0.153 & 0.112 & 0.231 \\
\hline ACOPF & 0.012 & 0.123 & 0.098 & 0.212 \\
\hline
\end{tabular}


What is interesting about the data in this table is that for $\lambda \geq 0.31$ the $\alpha$ value has a constant value. This result was anticipated. However, the reason for this is probably that for $\lambda \geq 0.31$, VRD reaches its control limits, thus, its effects have been reduced. Fig.4. presents impact of $\lambda$ value on number of switchable lines, i.e., $\eta$ value. What is interesting about the data in this table is that the $\eta$ value can be decreased by increasing the $\lambda$ value. Thus, it is apparent from this table that that performance of compensation level, may affect the TS action. It is, therefore, necessary to cooptimization TS action in the VRD control, in order to ensure that maximum performance will be achieved.

\section{Impact of Number of Switchable Lines}

Fig. 5 shows the effect of increasing the $\eta$ value on $\alpha$ value. As shown in Fig.5, the $\alpha$ value is increased by increases the $\eta$ value. This result expected, because in this power system seven transmission lines are congested, and also, the system congestion is the main obstacle to increase system's level of robustness. In this way, the TS action by open more transmission lines can more mitigate system congestion. From the data in Fig.5, it is apparent that for $\eta \geq 5$, no increase in the $\alpha$ value is detected. However, for $\eta \geq 5$ the system congestion is fully removed.

\section{Impact of Power Flow model on Simulation Results}

In this case, the solution results that obtained from DCOPF compare with ACOPF results. According to Table 1 and Figs. 3 and 5, some observations can be made. First, $\alpha$ value for ACOPF model in Table 1 and Figs. 3 and 5, is comparatively smaller than that of the $\alpha$ value for DCOPF model. Also, the $\eta$ value for DCOPF model in Fig. 4 is comparatively higher than that of the $\eta$ value for DCOPF model, where voltage magnitude constraints are not tightly enforced. These differences are due to constraints not modeled in the DCOPF model i.e., reactive power and voltage magnitude constraints.

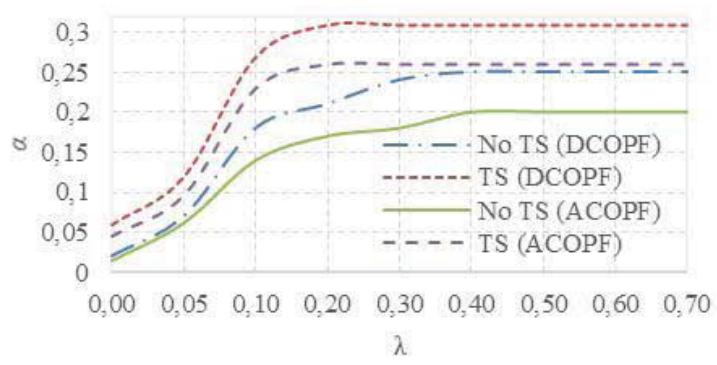

Fig. 3. The $\alpha$ value as a function of the $\lambda$ value; $\lambda=7$.

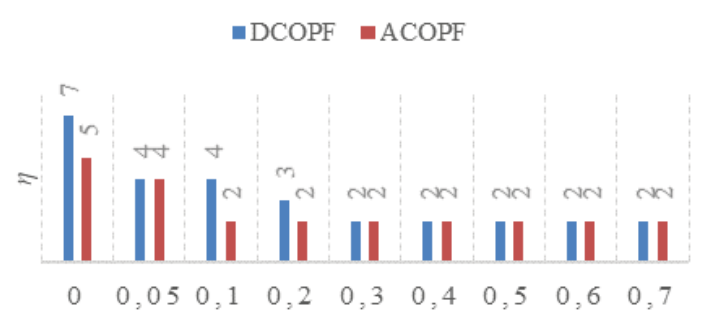

$\lambda$

Fig.4. The $\eta$ value as a function of the $\lambda$ value.

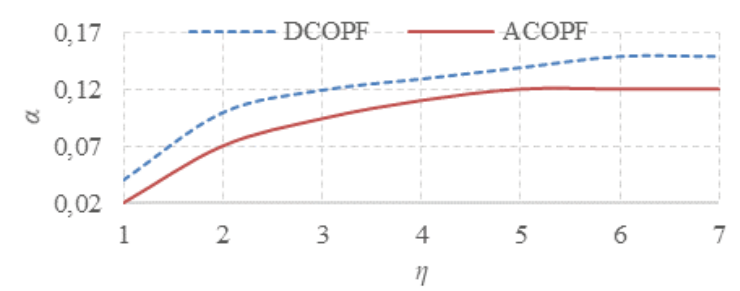

Fig.5. Optimal solution of $\alpha$ value under different the $\eta$ value; $\lambda=0.15$

\section{CONCLUSIONS}

This paper provides a technique to find worst-case wind uncertainty in an ACOPF model using an interval-based robust approach. TS and VRDs are incorporated as PFCTS for a more efficient utilization of existing transmission grid infrastructures. TS actions and VRD were found to be able to cooperatively offer significant capability in controlling worst-case wind uncertainty. However, it was shown that an ACOPF formulation with PFCTs would result in an MINLP. Accordingly, BDA was used to solve the MINLP problem. The simulation results proved that the BDA was able to find the optimal solution for a modified RTS-96 test system.

\section{REFERENCES}

[1] Smart Wires Technology,\| Smart Wires Inc. [Online]. Available: http://www.smartwires.com/technology/.

[2] O. Ziaee and F. F. Choobineh, "Optimal location-allocation of TCSC devices on a transmission network," IEEE Trans. Power Systems, vol. 32, no. 1, pp. 94-102, 2017.

[3] A. Nasri, A. J. Conejo, S. J. Kazempour, and M. Ghandhari, "Minimizing wind power spillage using an OPF with FACTS devices," IEEE Trans. Power Systems, vol. 29, no. 5, pp. 2150-2159, 2014.

[4] J. Aghaei, A. Nikoobakht, M. Mardaneh, M. Shafie-khah, and J. P. Catalão, "Transmission switching, demand response and energy storage systems in an innovative integrated scheme for managing the uncertainty of wind power generation," International Journal of Electrical Power \& Energy Systems, vol. 98, pp. 72-84, 2018.

[5] A. Nikoobakht and J. Aghaei, "IGDT-based robust optimal utilisation of wind power generation using coordinated flexibility resources," IET Renewable Power Generation, vol. 11, no. 2, pp. 264-277, 2016.

[6] K. W. Hedman, M. C. Ferris, R. P. O'Neill, E. B. Fisher, and S. S. Oren, "Co-optimization of generation unit commitment and transmission switching with N-1 reliability," IEEE Trans. Power Systems, vol. 25, no. 2, pp. 1052-1063, 2010.

[7] A. Nikoobakht, J. Aghaei, and M. Mardaneh, "Securing highly penetrated wind energy systems using linearized transmission switching mechanism," Applied Energy, vol. 190, pp. 1207-1220, 2017.

[8] A. Khodaei and M. Shahidehpour, "Transmission switching in securityconstrained unit commitment," IEEE Trans. Power Systems, vol. 25, no. 4, pp. 1937-1945, 2010.

[9] Y. Sang and M. Sahraei-Ardakani, "The interdependence between transmission switching and variable-impedance series FACTS devices," IEEE Trans. Power Systems, vol. 33, no. 3, pp. 2792-2803, 2017.

[10] W. Zheng, W. Wu, B. Zhang, and Y. Wang, "Robust reactive power optimisation and voltage control method for active distribution networks via dual time-scale coordination," IET Generation, Transmission \& Distribution, vol. 11, no. 6, pp. 1461-1471, 2017.

[11] C. Lee, C. Liu, S. Mehrotra, and Z. Bie, "Robust distribution network reconfiguration," IEEE Trans. Smart Grid, vol. 6, no. 2, pp. 836-842, 2014.

[12] A. Nikoobakht, J. Aghaei, H. Farahmand, V. Lakshmanan, and M. Korpås, "Flexibility of controllable power transformers for managing wind uncertainty using robust adjustable linearised optimal power flow," IET Renewable Power Generation, vol. 13, no. 2, pp. 262-272, 2018.

[13] A. Nikoobakht, J. Aghaei, T. Niknam, M. Shafie-khah, and J. P. Catalão, "Smart Wire Placement to Facilitate Large-Scale Wind Energy Integration: An Adaptive Robust Approach," IEEE Trans. Sustainable Energy, 2018.

[14] B. Zeng and L. Zhao, "Solving two-stage robust optimization problems using a column-and-constraint generation method," Operations Research Letters, vol. 41, no. 5, pp. 457-461, 2013.

[15] R. T. Force, "The IEEE reliability test system-1996," IEEE Trans. Power Syst., vol. 14, no. 3, pp. 1010-1020, 1999. 